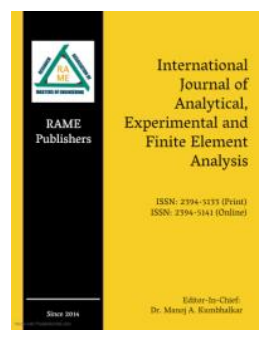

Pranav Pachpande pranav2641999@gmail.com

S. A. Karve

shivakarve@gmail.com

Department of Mechanical

Engineering,

JSPM Narhe Technical

Campus, Pune-411041,

Maharashtra, India

\title{
Magnetic Refrigeration: The Modern Refrigeration Technique- A Review
}

Abstract - This paper involves the information about type of newly refrigeration. The aim of this study is to give the working principle, operating cycle of the cooling due to the magnetic field. The aim behind the cooling effect is Magneto-Caloric effect MCE. According to this effect when magnetic material like gadolinium is subjected to field developed due to the magnet, temperature of that material increases and when source to develop the magnetic field is removed it returns to its normal temperature. The cooling effect caused uses the magnetic effect in the various ways. Gadolinium is kept as it will pass through magnetic field. As it transfers through the magnetic field the gadolinium heats up as it enters the magneto-caloric effect. There is need to circulate the cooled water to remove the heat out of the metal when it is in magnetic field. As the material lives the source of field, the materials decreases its temperature down its original temperature as the result of magnetic effect. Then this cold gadolinium is used to remove the heat from the refrigerator coils.

Index Terms - Magnetic refrigeration, regenerator, magnetic field, gadolinium.

\section{INTRODUCTION}

The vapor compression process is a mostly used in household refrigerator, air conditioners and many large industrial uses. However human being is facing serious crises because of global environment and spread of the gasses of fluid refrigerant such as CFC, HCFC, HFC are responsible for global warming. To overcome this problem, eco-friendly refrigerant are required or the new technology is required.

Refrigeration depended on the magnetic effect is the modern method to decrease temperature. This technology is ecofriendly because as the old technology independent work on expansion and compression of the sources (gasses), it never uses the hazardous gasses which leads to greenhouse effect. Magnetic refrigeration has a high efficiency like commonly used appliances [1].

Review Article

Published online - 10 April 2020

This is an open access article under the CC BY 4.0 International License https://creativecommons.org/licenses/by/4.0/

Cite this article - Pranav Pachpande and S. A. Karve, "Magnetic refrigeration: The modern refrigeration technique - A Review," International Journal of Analytical, Experimental and Finite Element Analysis, RAME Publishers, vol. 7, issue 1, p. 1, April 2020. https://doi.org/10.26706/ijaefea.1.7.20200303
Reliable refrigeration technique is the most important uses of the modern society. Unknown from the technique, food supply will be nonregular and limited to normally produced nonperishable items. Also the human comfort would be impossible [2].

Worldwide, about $15 \%$ of the overall energy consumption is from the refrigeration. Most modern refrigeration units are depend on the vapors compression cycle, whose development is strictly related to the effects of working fluid, i.e. CFCs and HFCs, have been because of their harmful effect and leading to global warming [3]. AMRs which perform as combined heat storage, heat exchanger and thermal energy generator are moving closer to a possible commercialization, as they can increases the temperature time many times the temperature change due to addition of MCM [4].

The MCM geometry has critical factors that affect the AMR performance. The cause behind is that a required porous structure of the MCM can be effectively transfer current to the thermal energy at thermal reservoir operating over useful temperature span [5]. Packed bed regenerators are most widely used geometry in AMRs is because of its high cooling performance and easy fabrication. The reason 
that other geometries such as: (1) thin walls are needed to facilitate heat condition from interior to the area occupied of MCM due to finite heat transfer [6].

(2) Thin walls are difficult to fabricate due to insufficient mechanical strength in MCMs [7].

(3) high thermal efficiency require small flow channel thickness, which can be difficult to manufacture consistency [8]. The opaque character of the MCM should not be too high to keep mechanical power constant and energy generation density of the generator to reproduce. Consequently, thin wall geometry results in a small hydraulic diameter and thus high flow resistance [6].

The characteristic diagram is even a more difficult for the case of $\mathrm{Nd}_{0.5} \mathrm{Sr}_{0.5} \mathrm{MnO}_{3}$ as it has been study and said that there is a presence of an A-type anti-ferromagnetic [9]-[12] and that there is also a presence of antiferromagnet [11], [13], [14].

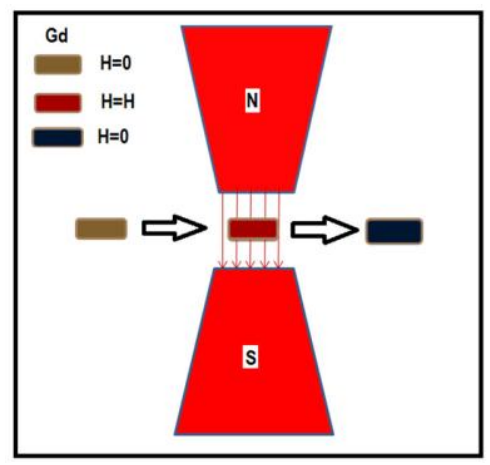

Figure 1. Gadolinium alloy inside the magnetic field and low temperature output [15]

\section{REGENERATOR PREPARATION AND GEOMETRIC CHARACTERIZATION}

Three regenerators as appeared in figure $2 \mathrm{~A}, \mathrm{~B}, \mathrm{C}$ were manufactured by freeze throwing under the comparable conditions to the past investigation of Liang et. A1. [6] on freeze cast regenerators i.e. figure 2 E. A ceramic suspension for freeze throwing was set up from 30vol\% of LCSM06 (CerPoTech , Norway) in MiliQ water with 2.5 $\mathrm{wt} \%$, solid to ceramic ratio, of disperent (DURAMAX^TM 3005, Rohmand Haas, Dow Chemical, USA). PH was changed in accordance with 7 by expansion of $1 \mathrm{M}$ nitric corrosive to build up a sufficient scattering of particles. The suspension was then homogenized for $72 \mathrm{~h}$ on a low vitality ball plant. $2 \mathrm{wt} \%$, strong to fired proportion, of folio (DURAMAXTM B-1022, Rohm and Haas, Dow Chemical, USA), was included and the suspension was blended for a couple of hours. The suspension air is taken out in vacuum preceding throwing. During freeze-throwing, the suspension of LCSM06 particles in water was solidified directionally by bringing one side of the suspension into contact with a cooling source. Here, we have utilized the freeze throwing arrangement with the control of thermoelectric temperature of the accompanying source [6].

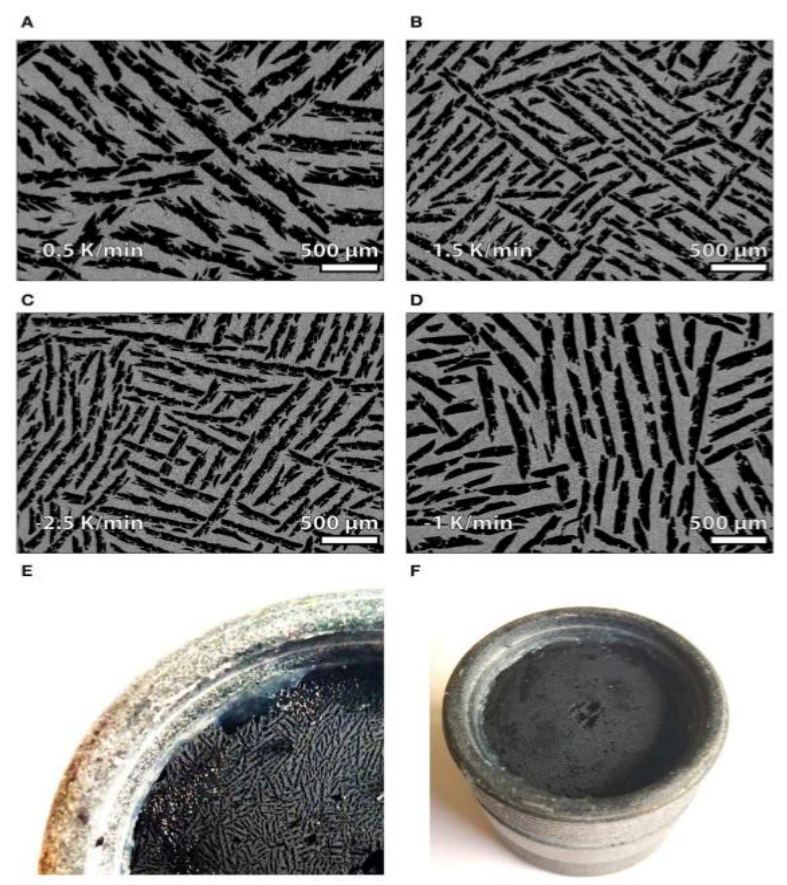

Figure 2. Geometry of freeze cast regenerator [6]

The cooling source is diminished by $-0.5,-1.5$ and -2.5 $\mathrm{K} / \mathrm{min}[6]$ and make the raising temperature inclination to cause the ice structure towards the slope course, pushing aside particles, causing these to isolate, which brings about a two stage structure of solidified water precious stone along the warm angle and isolated artistic particles. The ice is taken out by sublimation in a freeze-drier and the green bodies were sintered at $1100{ }^{\circ} \mathrm{C}$ for $12 \mathrm{~h}$, bringing about permeable earthenware structures with well-defined microchannels where the ice was at that point. The state of the solidified water (precious stone) while freeze-throwing, and in this manner the shape coming about miniaturized scale directs in the fired earthenware, depends on the 
freezing conditions; where quicker cooling rates brings about littler channels [16]. Here, just the freezing rates were changed regarding accomplish a scope of pore widths empowering the investigation of the influence of pore width on AMR execution. The three new regenerators are contrasted and our recently distributed regenerator (\#4), and all the geometrical parameters are summed up in Table 1. The auxiliary highlights of the freeze-cast regenerators were described by investigation of figure of micrographs, is clarified in detail in past examinations [17]. Micrographs were acquired utilizing an exceptional examining magnifying instrument (TM3000, Hitachi HighTechnologies). For each example 12 micrographs, covering a region of $3310 \times 2483 \mu \mathrm{m}$ each, in the opposite cross segment, got uniformly appropriated over the cross areas, were investigated. Pore width, full scale porosity, specific surface territory and tortuosity were assessed relies upon figure analysis. From the regenerators with little pore width to enormous pore width (Figures 2 A-D), the necessary proportion of cross sectional pore shape is increments. That implies the freeze cast generators which recover with the little pore width are inclined to have thin channels. As freeze-throwing is exceptionally delicate to obvious conditions convoluting the reproducibility of freeze-throws across different suspension clusters, set-ups, heater conditions and so on [18], A-C were manufactured from a similar group, solidified in this way and dried and sintered together. As needs be, regenerator A-C shows a bigger level of dendrites blocking the channels than regenerator $\mathrm{D}$, which was created from a different group and with much smoother channel dividers. In permeable media, the pores can be interconnected, impasse or isolated [15]. The all out void volume partitioned by the all out volume involved by the strong framework and voids volumes, is defined as the complete porosity, or test porosity $(\varepsilon)$ in this investigation. In any case, the fluid just flows through the interconnected pores. The volume portion of the interconnected pores is defined as the effective porosity or full scale porosity $(\mathrm{cm})$, which is gotten from picture investigation. As observed from Table 1the Macro porosity is around 2/3 of the all out porosity. In this way, the impasse pores are expected irrelevant. The rest of the porosity can be treated as small scale porosity in the dividers. In this examination, $\varepsilon_{\mathrm{m}}$ is utilized for deciding pore speed while $\varepsilon$ is received for strong mass estimations. Furthermore, the pressure driven measurement $\left(\mathrm{D}_{\mathrm{h}}\right)$ and tortuosity $(\mathrm{T})$ are determined as follows:

$$
\begin{aligned}
& \mathrm{D}_{\mathrm{h}}=4 \varepsilon_{\mathrm{m}} / \alpha \\
& \mathrm{T}=\mathrm{L}_{\text {pore }} / \mathrm{L}_{\mathrm{r}}
\end{aligned}
$$

where $\alpha$ is the specific surface area derived from image analysis, $\mathrm{L}_{\text {pore }}$ is the average pore length, and $\mathrm{L}_{\mathrm{r}}$ is the length of the regenerator sample [6]. The heat exchange fluid enters the wheel at a temperature Thot, and leaves the wheel at a temperature $\mathrm{T}_{\text {cold }}$ after expels heat to the magnetic refrigerant in low-field. Then, the fluid re-enters the wheel at temperature $\mathrm{T}_{\text {cold }}+\Delta$ after picking up refrigeration heat load $\mathrm{Q}_{\text {cold. }}$. In the thermal exchange with the wheel which is at temperature $\mathrm{T}_{\text {hot }}+\Delta$, the fluid warms to $\mathrm{T}_{\text {hot }}+\Delta$.

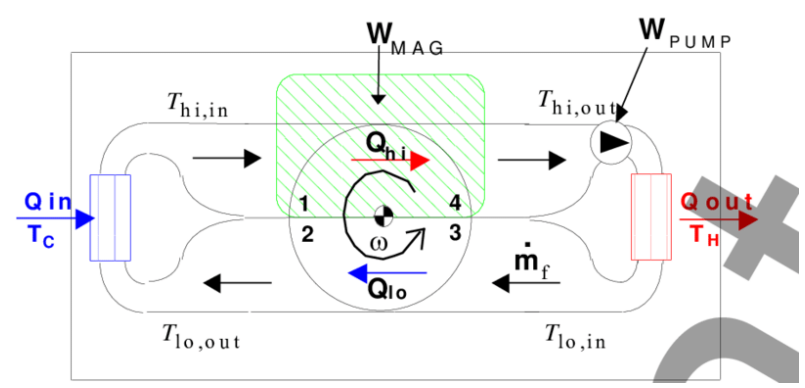

Figure 3. Steyert magnetic refrigeration [19]

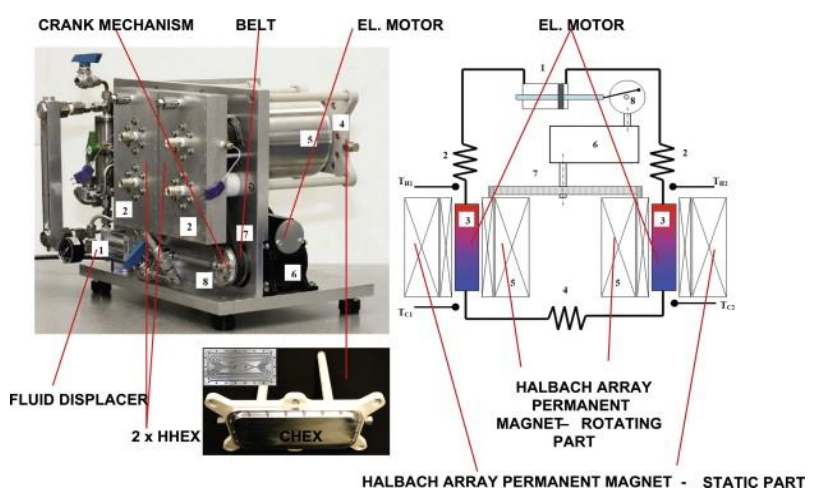

Figure 4. Kirol system [20]

\section{A. Magnetic Refrigeration Materials}

Since Brown previously applied ferromagnetic material gadolinium $\left(\mathrm{G}_{\mathrm{d}}\right)$ in the room temperature attractive cooler 
in 1976, the examination run for attractive refrigeration working materials has been incredibly extended. From the outset, some ferromagnets concerning the subsequent request progress were researched for the huge MCE existing in them. As of late the attractive materials experiencing a first-request attractive change become the concentration after the monster MCE was found in GdSiGe amalgams. The magneto-caloric impact is an inherent property of attractive strong. This impact is obeyed by all change metals and lanthanide-arrangement components. The initially recommended refrigerant was a paramagnetic salt, for example, cerium magnesium nitrate. Gadolinium, an uncommon earth metal, shows one of the biggest known MCE. It was utilized as a refrigerant for a significant number of the early attractive refrigeration structure.

Roman images for amounts and factors, yet not Greek images. Accentuate conditions with commas or periods when they are a piece of a sentence [20].

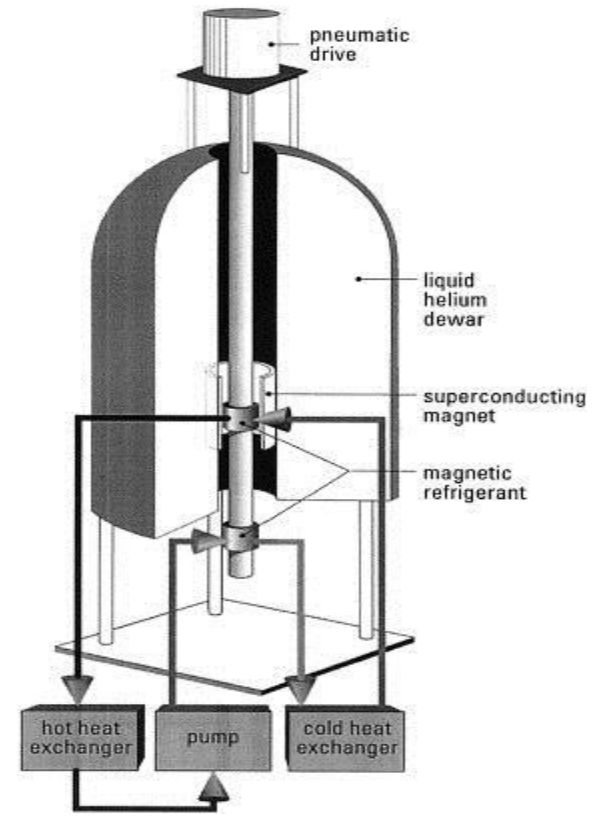

Figure 5. Zimm refrigeration system [21]

\section{CRITERIA FOR SELECTING ROOM TEMPERATURE}

\section{MATERIAL}

Contingent upon the relating hypothetical investigation the attribute of MCE, magnetic materials in magnetic refrigeration should finish a few highlights for utilizing [22], i. The large deltaSM and deltaT

ii. The large density of magnetic entropy, which decide the working efficiency of materials

iii. The small lattice entropy

iv. The modest curie temperature in the vicinity room to guarantee the magnetic entropy change can be obtained in a whole temperature vary of the process

v. The nearly zero magnetic hysteresis

vi. The very small thermal hysteresis

vii. The small specific heat and large thermal conductivity

viii. The more electric resistance

ix. It should have high chemical stability

First-order phase transitions in magnetic materials have gained strong interest, due to their potential applicability in magnetic refrigeration at room temperature [23]. Magnetic refrigeration is based on the magnetocaloric effect (MCE), which is defined as heating or cooling of a magnetic material in a changing magnetic field [24], [25]. The MCE is determined quantitatively in terms of the isothermal entropy change or the adiabatic temperature change [25]. A giant MCE around room temperature was first discovered in $\operatorname{Gd} 5(\mathrm{Si1}-\mathrm{xGex})$ [26], [27]. Following this discovery, a few other systems, such as LaFe13-xSix [28], [29], MnAs1-xSbx [30], and MnFeP1-xAsx [31], were obtained a giant MCE [31]. Recently, considerable attention has been paid to the $\mathrm{Mn}$ rich $\mathrm{Ni} 2-\mathrm{xMn} 1+\mathrm{xZ}(\mathrm{Z}=\mathrm{Sn}$, In, Sb) based magnetic shape memory Heusler alloys that undergo a first-order diffusion less martensitic phase transformation from a high-temperature high-symmetry cubic austenite phase to a low-temperature low-symmetry martensitic phase, which can have tetragonal, orthorhombic, or monoclinic symmetry [32], [33]. The first-order martensitic phase transition, driven by nucleation and growth of the austenite phase, leads to many great properties, such as shape memory, magnetic super elasticity, and caloric effects [33], [34].The origin of these physical properties is coming from the strong interrelation between crystal structure and magnetism. Specifically, the crystallographic 
change at the martensitic transition can generate a large MCE usable in cooling applications [33], [35]. Among the $\mathrm{Ni}_{2}-x \mathrm{xn}_{1}+\mathrm{xZ}\left(\mathrm{Z}=\mathrm{S}_{\mathrm{n}}, \mathrm{I}_{\mathrm{n}}, \mathrm{S}_{\mathrm{b}}\right)$ Heusler alloys, the In-based ones are the assured in terms of magnetic refrigeration due to the significant cooling effect with reversible adiabatic change, $\mathrm{T}_{\mathrm{ad}}$, of upto 5.4K [13][20]. However, the large $\mathrm{T}_{\mathrm{ad}}$ cannot be observed in successive field cycles due to the large thermal hysteresis in these materials [36], [37]. Therefore, nowadays most of the efforts are devoted to reduce the hysteresis aiming at a reversible MCE. The thermal hysteresis can be reduced by different methods, such as chemical pressure by doping of an appropriate element, tuning the composition, annealing conditions, or physical pressure. As a result interatomic distances change which leads to a modification of magnetic interactions [38], [39]. However, the methods used for a reduction of the hysteresis affect not only the thermal hysteresis, but also the magnetic properties which include transition temperatures, sharpness of the transition and, thus, the magnetocaloric properties. That makes the implementation of this promising strategy tricky in Heusler alloys [40][42].

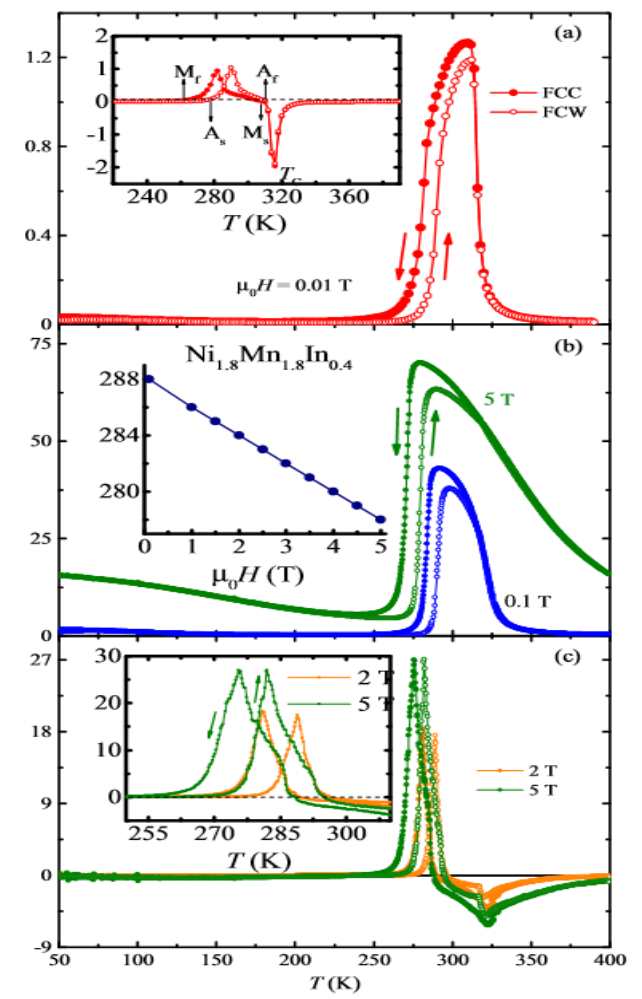

Fig 6. (a) Field cooled cooling and Field cooled warming magnetization $\mathrm{M}(\mathrm{T})$ curves at $0.01 \mathrm{~T}$ for $\mathrm{Ni}_{1.8} \mathrm{Mn}_{1.8} \mathrm{In}_{0.4} . \mathrm{D}_{\mathrm{m}}(\mathrm{T}) / \mathrm{D}_{\mathrm{t}}$ is shown in inset. (b) $\mathrm{M}(\mathrm{T})$ curves at different magnetic field of 0.1 and 5T. The inset shows the shift of martensitic transition temperature Tm as the work of the field due to magnetic effect

(c) Isothermal magnetic entropy change $\mathrm{SM}(\mathrm{T})$. calculated from the corresponding $\mathrm{M}(\mathrm{T})$ curves on the heating and cooling. The inset present $\mathrm{SM}(\mathrm{T})$ on an expanded scale around the martensitic transition. FCC and FCW data are represented by rigid and unclosed symbols, respectively

[36].

\section{A. Mathematical concept}

It's well known that in paramagnetic region the relation between $\mathrm{X}$ and temperature $\mathrm{T}$ should follow the curie-weiss law. This is given by the following

$$
\mathrm{X}=\mathrm{C} / \mathrm{T}-\theta \mathrm{WC}
$$

Where $\theta_{\mathrm{P}}$ is the Weiss temperature and $\mathrm{c}$ is the curie constant defined as;

$$
\mathrm{C}=\mathrm{NA} \mu 2 \mathrm{~B} / 3 \mathrm{~KB} \times \mu 2
$$

where NA $=6.023 \times 1023 \mathrm{~mol}^{-1}$ is the number of Avogadro; $\mu_{\mathrm{B}}=9.274 \times 10-24\left(\mathrm{Am}^{2}\right)$ is the Bohr magneton; and $\mathrm{k}_{\mathrm{B}}=1.38016 \times 10-23 \mathrm{~J} \mathrm{~K}^{-1}$ is the Boltzmann constant From fitting the linear paramagnetic region, Curie-Weiss $\mathrm{C}$ and $\theta_{\mathrm{P}}$ parameters were obtained. The positive $\theta_{\mathrm{P}}$ value indicates the presence of a ferromagnetic interaction between the spins. From the determined $\mathrm{C}$ parameter, we have deduced the $\mu_{\text {expleff }}$ values. As summing orbital momentum to be quenched in $\mathrm{Nd}^{3+}, \mathrm{Sr}^{2+}, \mathrm{Mn}^{3+}$ and $\mathrm{Mn}^{4+}$ the theoretical paramagnetic effective moment can be written as:

where $\mathrm{g}=1+\mathrm{J}(\mathrm{J}+1)+\mathrm{S}(\mathrm{S}+1)-\mathrm{L}(\mathrm{L}+1) 2(\mathrm{~J}(\mathrm{~J}+1)$ is the Landé factor; $\mathrm{J}=|\mathrm{S}+\mathrm{L}|$, total moment; $\mathrm{L}=\sum \mathrm{ml}$, orbital moment; and $\mathrm{S}=\sum \mathrm{ms}$, spin moment. The values of total moment, orbital moment, spin moment and effective moment of species present in this compound are listed in Table 1. The theoretical paramagnetic effective moment can be written as

Table 2 summarizes the temperature dependence of $T_{C}, \theta_{P}$, $\mu_{\text {thleff }}$ and $\mu_{\text {expleff }}$ for an applied magnetic field of $0.05 \mathrm{~T}$. Thus, we notice an agreement between the theoretical value of the magnetic moment and the value expected for an isolated paramagnetic system. This result shows that for a field of $0.05 \mathrm{~T}$, all spins are aligned. 
TABLE I

VALUE OF TOTAL MOMENT, ORBITAL MOMENT, SPIN MOMENT, EFFECTIVE MOMENT

\begin{tabular}{|c|c|c|c|c|c|}
\hline & $\mathrm{A}$ & $\mathrm{B}$ & $\mathrm{C}$ & $\mathrm{D}$ & $\mathrm{E}$ \\
\hline $\mathrm{Nd}^{3+}$ & $3 / 2$ & 6 & $9 / 2$ & 1.33 & 6.61 \\
\hline $\mathrm{Sr}^{2+}$ & 0 & 0 & 0 & 0 & - \\
\hline $\mathrm{Mn}^{3+}$ & 2 & 2 & 0 & 1 & 4.90 \\
\hline $\mathrm{Mn}^{4+}$ & $3 / 2$ & 3 & $3 / 2$ & $2 / 5$ & 3.87 \\
\hline
\end{tabular}

TABLE II

TRANSITION TEMPERATURE $\left(\mathrm{T}_{\mathrm{C}}, \mathrm{T}_{\mathrm{G}}, \Theta_{\mathrm{P}}, \mathrm{T}_{\mathrm{R}}\right.$ AND C), THE EFFECTIVE MOMENT EXPERIMENTAL AND THEORETICAL

\begin{tabular}{|c|c|}
\hline $\mathrm{Nd}_{0.6} \mathrm{Sr}_{0.4} \mathrm{MnO}_{3}$ & 245 \\
\hline $\mathrm{T}_{\mathrm{c}}(\mathrm{K})$ & 270 \\
\hline $\mathrm{T}_{\mathrm{G}}(\mathrm{K})$ & 245 \\
\hline$\Theta_{\mathrm{p}}(\mathrm{k})$ & 130 \\
\hline $\mathrm{T}_{\mathrm{SG}}(\mathrm{K})$ & \\
\hline$\mu_{\text {exp/eff }}$ & 6.98 \\
\hline$\mu_{\text {thleff }}$ & \\
\hline $\mathrm{T}_{(\text {Rand/c) }} \mathrm{k}$ & 6.82 \\
\hline
\end{tabular}

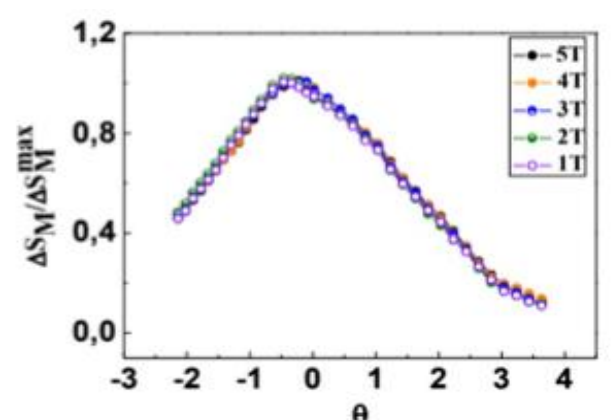

Fig 7. Normalized entropy change versus rescaled temperature for different applied magnetic field [39]

\section{Conclusions}

In this work we have examined the output of the magnetic refrigeration and its uses as the modern technique instead of the traditional which leads to the global warming. Finally with the help of the calculation and practical, a detailed study of magnetic and magneto caloric properties has been conducted. The given information tells that is a theoretical model useful for the study of refrigeration due to magnetic effect, constructing a refrigerator model we can predict the values of the magnetic change full-width at half-maximum.

\section{REFERENCES}

[1] C. Zimm, J. Auringer, A. Boeder, J. Chell, S. Russek, and A. Sternberg, 'Design and initial performance of a magnetic refrigerator with a rotating permanent magnet', Proc.Int. Conf. Magn. Refrig. Room Temp. Portoroz, Slov., no. April, pp. 341-347, 2007, doi: 10.4065/mcp.2010.0817.

[2] V. K. Pecharsky, J. Cui, and D. D. Johnson, '(Magneto)caloric refrigeration: is there light at the end of the tunnel?', Philos. Trans. R. Soc. A Math. Phys. Eng. Sci., vol. 374, no. 2074, p. 20150305, Aug. 2016, doi: 10.1098/rsta.2015.0305.

[3] C. Aprea, A. Greco, A. Maiorino, and C. Masselli, 'Magnetic refrigeration: An eco-friendly technology for the refrigeration at room temperature', in Journal of Physics: Conference Series, 2015, vol. 655, no. 1, doi: 10.1088/17426596/655/1/012026.

[4] A. Kitanovski and P. W. Egolf, 'Thermodynamics of magnetic refrigeration', International Journal of Refrigeration, vol. 29, no. 1. Elsevier, pp. 3-21, Jan. 01, 2006, doi: 10.1016/j.ijrefrig.2005.04.007.

[5] Y. Lei, K. Liu, L. Hou, L. Ding, Y. Li, and L. Liu, 'Small chaperons and autophagy protected neurons from necrotic cell death', Sci. Rep., vol. 7, no. 1, pp. 1-13, Dec. 2017, doi: 10.1038/s41598-017-05995-6.

[6] J. Liang, C. D. Christiansen, K. Engelbrecht, K. K. Nielsen, R. Bjørk, and C. R. H. Bahl, 'Characterization of Freeze-Cast Micro-Channel Monoliths as Active and Passive Regenerators', Front. Energy Res., vol. 8, no. April, 2020, doi: 10.3389/fenrg.2020.00054.

[7] B. Monfared and B. Palm, 'Material requirements for magnetic refrigeration applications', Int. J. Refrig., vol. 96, pp. 25-37, Dec. 2018, doi: 10.1016/j.ijrefrig.2018.08.012.

[8] K. K. Nielsen, K. Engelbrecht, and C. R. H. Bahl, 'The influence of flow maldistribution on the performance of inhomogeneous parallel plate heat exchangers', Int. J. Heat Mass Transf., vol. 60, no. 1, pp. 432-439, May 2013, doi: 10.1016/j.ijheatmasstransfer.2013.01.018.

[9] R. Kajimoto et al., 'Hole-concentration-induced transformation of the magnetic and orbital structures in $\mathrm{Nd1-}$ xSrxMnO3', Phys. Rev. B - Condens. Matter Mater. Phys., vol. 60, no. 13, pp. 9506-9517, Oct. 1999, doi: 10.1103/PhysRevB.60.9506.

[10] H. Kawano, R. Kajimoto, H. Yoshizawa, Y. Tomioka, H. Kuwahara, and Y. Tokura, 'Magnetic ordering and relation to the metal-insulator transition in $\operatorname{Pr} 1-\mathrm{xSrxMnO} 3$ and $\mathrm{Nd} 1-$ 
xSrxMnO3 with $\mathrm{x} \sim 1 / 2$ ', Phys. Rev. Lett., vol. 78, no. 22, pp. 4253-4256, Jun. 1997, doi: 10.1103/PhysRevLett.78.4253.

[11] C. Ritter and R. Mahendiran, 'Direct evidence of phase segregation and magnetic-field-induced structural transition in by neutron diffraction', Phys. Rev. B - Condens. Matter Mater. Phys., vol. 61, no. 14, pp. R9229-R9232, Apr. 2000, doi: 10.1103/PhysRevB.61.R9229.

[12] J. P. Joshi, A. K. Sood, S. V. Bhat, S. Parashar, A. R. Raju, and C. N. R. Rao, 'An electron paramagnetic resonance study of phase segregation in $\mathrm{Nd} 0.5 \mathrm{Sr} 0.5 \mathrm{MnO} 3$, J. Magn. Magn. Mater., vol. 279, no. 1, pp. 91-102, Aug. 2004, doi: 10.1016/j.jmmm.2004.01.072.

[13] V. T. Dovgii et al., 'Anomalous magnetic susceptibility in $\mathrm{Nd} 0.5 \mathrm{Sr} 0.5 \mathrm{MnO} 3$ manganite single crystals', Tech. Phys. Lett., vol. 34, no. 12, pp. 1044-1046, Dec. 2008, doi: 10.1134/S106378500812016X.

[14] J. Geck et al., 'Anisotropic CE-type orbital correlations in the ferromagnetic metallic phase of (formula presented)', Phys. Rev. B - Condens. Matter Mater. Phys., vol. 66, no. 18, pp. 18, Nov. 2002, doi: 10.1103/PhysRevB.66.184407.

[15] I. A. Abdel-Latif and M. R. Ahmed, 'Use of Magnetocaloric Material for Magnetic Refrigeration System: A Review', Mater. Sci. Res. India, vol. 16, no. 3, pp. 209-224, 2019, doi: $10.13005 / \mathrm{msri} / 160303$.

[16] S. E. Naleway, J. R. A. Taylor, M. M. Porter, M. A. Meyers, and J. McKittrick, 'Structure and mechanical properties of selected protective systems in marine organisms', Materials Science and Engineering C, vol. 59. Elsevier Ltd, pp. 11431167, Feb. 01, 2016, doi: 10.1016/j.msec.2015.10.033.

[17] M. Kaviany, 'Principles of Heat Transfer in Porous Media', Mech. Eng. Ser., vol. 53, no. 9, p. 726, 1995, doi: 10.1007/978-1-4612-4254-3.

[18] C. Zimm et al., 'Description and Performance of a NearRoom Temperature Magnetic Refrigerator', in Advances in Cryogenic Engineering, Springer US, 1998, pp. 1759-1766.

[19] L. A. Tagliafico, F. Scarpa, F. Canepa, and S. Cirafici, 'Performance analysis of a room temperature rotary magnetic refrigerator for two different gadolinium compounds', Int. J. Refrig., vol. 29, no. 8, pp. 1307-1317, 2006, doi: 10.1016/j.ijrefrig.2006.07.017.

[20] B. R. Dorin, J. Avsec, and A. Plesca, 'The Efficiency Of Magnetic Refrigeration And A Comparison With Compressor Refrigeration Systems', 2018. Accessed: May 26, 2020. [Online]. Available: www.fe.um.si/en/jet.html.

[21] M. Almanza, A. Kedous-Lebouc, J. P. Yonnet, U. Legait, and
J. Roudaut, 'Magnetic refrigeration: Recent developments and alternative configurations', EPJ Appl. Phys., vol. 71, no. 1, 2015, doi: 10.1051/epjap/2015150065.

[22] A. P. Garole, A. B. More, and G. P. Jarad, "'Analysis of Factors Influencing Time Overrun in Build Operate Transfer Infrastructure Projects: A Case Study on BOT Road Project in Maharashtra"”, Int. Res. J. Eng. Technol., 2016, Accessed: May 26, 2020. [Online]. Available: www.irjet.net.

[23] V. Franco, J. S. Blázquez, B. Ingale, and A. Conde, 'The Magnetocaloric Effect and Magnetic Refrigeration Near Room Temperature: Materials and Models', Annu. Rev. Mater. Res., vol. 42, no. 1, pp. 305-342, Aug. 2012, doi: 10.1146/annurev-matsci-062910-100356.

[24] E. Brück, 'Developments in magnetocaloric refrigeration', Journal of Physics D: Applied Physics, vol. 38, no. 23. 2005, doi: 10.1088/0022-3727/38/23/R01.

[25] A. Taubel et al., 'A Comparative Study on the Magnetocaloric Properties of Ni-Mn-X(-Co) Heusler Alloys', Phys. status solidi, vol. 255, no. 2, p. 1700331, Feb. 2018, doi: 10.1002/pssb.201700331.

[26] J. D. Moore et al., 'Metamagnetism Seeded by Nanostructural Features of Single-Crystalline $\mathrm{Gd}_{5} \mathrm{Si}_{2} \mathrm{Ge}_{2}$, Adv. Mater., vol. 21, no. 37, pp. 3780-3783, Oct. 2009, doi: 10.1002/adma.200900093.

[27] V. K. Pecharsky and K. A. Gschneidner, 'Giant magnetocaloric effect in Gd5 (Si2 Ge2)', Phys. Rev. Lett., vol. 78, no. 23, pp. 4494-4497, Jun. 1997, doi: 10.1103/PhysRevLett.78.4494.

[28] A. Fujita, S. Fujieda, Y. Hasegawa, and K. Fukamichi, 'Itinerant-electron metamagnetic transition and large magnetocaloric effects in (formula presented) compounds and their hydrides', Phys. Rev. B - Condens. Matter Mater. Phys., vol. 67, no. 10, p. 12, Mar. 2003, doi: 10.1103/PhysRevB.67.104416.

[29] J. Lyubina, K. Nenkov, L. Schultz, and O. Gutfleisch, 'Multiple metamagnetic transitions in the magnetic refrigerant La(Fe,Si)13Hx', Phys. Rev. Lett., vol. 101, no. 17, p. 177203 , Oct. 2008, doi: 10.1103/PhysRevLett.101.177203.

[30] H. Wada, K. Taniguchi, and Y. Tanabe, 'Extremely Large Magnetic Entropy Change of MnAs 1-x Sb x near Room Temperature', 2002.

[31] O. Tegus, E. Brück, K. H. J. Buschow, and F. R. De Boer, 'Transition-metal-based magnetic refrigerants for roomtemperature applications', Nature, vol. 415, no. 6868, pp. 150-152, Jan. 2002, doi: 10.1038/415150a. 
[32] Y. Sutou et al., 'Magnetic and martensitic transformations of $\mathrm{NiMnX}(\mathrm{X}=\mathrm{In}, \mathrm{Sn}, \mathrm{Sb})$ ferromagnetic shape memory alloys', in Applied Physics Letters, Nov. 2004, vol. 85, no. 19, pp. 4358-4360, doi: 10.1063/1.1808879.

[33] J. Liu, T. Gottschall, K. P. Skokov, J. D. Moore, and O. Gutfleisch, 'Giant magnetocaloric effect driven by structural transitions', Nat. Mater., vol. 11, no. 7, pp. 620-626, May 2012, doi: 10.1038/nmat3334.

[34] A. Planes, L. Mãosa, and M. Acet, 'Magnetocaloric effect and its relation to shape-memory properties in ferromagnetic Heusler alloys', J. Phys. Condens. Matter, vol. 21, no. 23, 2009, doi: 10.1088/0953-8984/21/23/233201.

[35] P. Devi et al., 'Adaptive modulation in Ni2Mn1.4In0.6 magnetic shape memory Heusler alloy', Phys. Rev. B, vol. 97, no. 22, Nov. 2016, doi: 10.1103/PhysRevB.97.224102.

[36] M. G. Zavareh et al., 'Direct measurements of the magnetocaloric effect in pulsed magnetic fields: The example of the Heusler alloy Ni\$_\{50\}\$Mn\$_\{35\}\$In\$_\{15\}\$, Appl. Phys. Lett., vol. 106, no. 7, Jan. 2015, doi: 10.1063/1.4913446.

[37] T. Gottschall, K. P. Skokov, B. Frincu, and O. Gutfleisch, 'Large reversible magnetocaloric effect in Ni-Mn-In-Co', Appl. Phys. Lett., vol. 106, no. 2, p. 021901, Jan. 2015, doi: 10.1063/1.4905371.
[38] L. Caron et al., 'Effect of Pt substitution on the magnetocrystalline anisotropy of Ni2MnGa: A competition between chemistry and elasticity', Phys. Rev. B, vol. 96, no. 5, p. 054105, Aug. 2017, doi: 10.1103/PhysRevB.96.054105.

[39] S. R. Barman et al., 'Theoretical prediction and experimental study of a ferromagnetic shape memory alloy: Ga2MnNi', Phys. Rev. B - Condens. Matter Mater. Phys., vol. 78, no. 13, p. 134406, Oct. 2008, doi: 10.1103/PhysRevB.78.134406.

[40] V. V. Khovaylo et al., 'Peculiarities of the magnetocaloric properties in Ni-Mn-Sn ferromagnetic shape memory alloys', Phys. Rev. B - Condens. Matter Mater. Phys., vol. 81, no. 21, p. 214406, Jun. 2010, doi: 10.1103/PhysRevB.81.214406.

[41] V. V. Khovaylo et al., 'Magnetic properties of Ni50 Mn34.8 In15.2 probed by Mössbauer spectroscopy', Phys. Rev. B Condens. Matter Mater. Phys., vol. 80, no. 14, p. 144409, Oct. 2009, doi: 10.1103/PhysRevB.80.144409.

[42] T. Gottschall et al., 'Dynamical Effects of the Martensitic Transition in Magnetocaloric Heusler Alloys from Direct $\Delta$ tad Measurements under Different Magnetic-Field-Sweep Rates', Phys. Rev. Appl., vol. 5, no. 2, p. 024013, Feb. 2016, doi: 10.1103/PhysRevApplied.5.024013. 